\title{
DIRECTRICES, LEGISLACIÓN Y PROMOCIÓN DE BIBLIOTECAS ESCOLARES EN ANDALUCÍA
}

\section{DIRECTIVES, LEGISLATION AND PROMOTION OF SCHOOL LIBRARIES IN ANDALUSIA}

\author{
Raúl Cremades García \\ Universidad de Málaga \\ cremades@uma.es \\ Concepción $M \underline{a}$ Jiménez Fernández \\ Revista Mi Biblioteca (www.mibiblioteca.org) \\ redaccion@mibiblioteca.org
}

Recibido: febrero, 2012.

Versión final aceptada: noviembre, 2012.

PALABRAS CLAVE: biblioteca escolar, legislación, recursos bibliotecarios, modelo de intervención, plataforma educativa.

KEYWORDS: school library, legislation, library resources, intervention model, educational platform.

\section{RESUMEN}

En este artículo se describen las principales directrices y legislación sobre biblioteca escolar vigentes en Andalucía y que han servido como punto de partida para promover estas bibliotecas en la comunidad andaluza. Además, se exponen las principales iniciativas de apoyo e impulso a la biblioteca escolar en Andalucía (Instituciones, encuentros y actividades de formación; Publicaciones periódicas; Planes, proyectos y redes; Recursos y herramientas) que juegan un papel protagonista en el desarrollo de estas instituciones.

\section{ABSTRACT}

The main current directives and legislation on school library in Andalusia are described in this article, those that have served as starting point to promote these libraries in the Andalusian community. In addition, there are exposed the principal initiatives of support and impulse to the school library in Andalusia (Institutions, meetings and formation activities; Periodic publications; Plans, projects and networks; Resources and tools) that play a leading role in the development of these institutions.

ISSN: 0212-8594 ISSN-e: 2340-2776 № DOI: http://dx.doi.org/10.12795/rea.2011.i28.02 


\section{INTRODUCCIÓN.}

A lo largo de las últimas décadas no han dejado de publicarse pautas y directrices diversas referidas al funcionamiento, características y objetivos de la biblioteca escolar. Pero, a pesar de ello, aún no existe un modelo común que englobe todos esos aspectos. Esto es debido a que nuestro país carece de tal modelo teórico que se adecue a la realidad sociocultural y educativa española, por lo que el concepto que se tiene de una biblioteca escolar es el de una institución sometida a permanentes debates y sin un concepto cerrado de lo que debe ser en realidad (Yaguez, E., 2006, p. 15).

En esa hipótesis abunda García, J. (2010, p. 32) cuando expone que en España las bibliotecas escolares se encuentran en un estado carencial y aunque en la última década se han producido mejoras, estas cambian de una comunidad autónoma a otra. El autor defiende la necesidad esencial de contar un modelo definido:

Un marco de referencia normativo que garantice estabilidad de recursos humanos, programas y tiempos para el funcionamiento de la biblioteca escolar, atendiendo a aspectos nucleares sin resolver, como responsables, instalaciones, equipamiento, fondos, presupuesto, horario de apertura y atención, coordinación con el resto de órganos del centro, equipos de apoyo, etcétera.

En este sentido, para la existencia de una biblioteca escolar solvente, la política se convierte en un elemento determinante. Por ello, las medidas legislativas y declaraciones generales relacionadas con estas bibliotecas tienen una importancia crucial, tanto para potenciar su uso como centro de recursos para la enseñanza y el aprendizaje como también para el proceso educativo en general. De la misma manera, y además de la normativa publicada en materia de bibliotecas, son esenciales todas aquellas iniciativas, tanto públicas como privadas, encaminadas a promover y apoyar esas bibliotecas escolares impulsando así su desarrollo y mayor visibilidad.

En este trabajo se exponen la legislación, directrices y programas de impulso y difusión de las bibliotecas escolares en Andalucía con el fin de proporcionar un marco de información sobre la planificación o rediseño de las bibliotecas en el ámbito andaluz.

\section{DIRECTRICES Y LEGISLACIÓN DE BIBLIOTECAS ESCOLARES EN ANDALUCÍA.}

Con miras a ajustarse al modelo descentralizado establecido por la Constitución española de 1978, la Administración del Estado se ha ido transformando hasta lograr una asunción de competencias en materia de educación por parte de las diferentes comunidades autónomas. Según esto, a las comunidades autónomas les corresponde el desarrollo de competencias ejecutivo-administrativas y de gestión del sistema

ISSN: 0212-8594 ISSN-e: 2340-2776 № DOI: http://dx.doi.org/10.12795/rea.2011.i28.02

REA 28 (2011):16-33

http://www.publius.us.es/estudios_andaluces 
educativo en su propio territorio. De esa manera, mediante Real Decreto 1075/1981, de 24 de abril (España 1981) se lleva a cabo el traspaso de competencias, funciones y servicios a la Junta de Andalucía en materia de Cultura. Del mismo modo, mediante Real Decreto 3936/1982, de 29 de diciembre (España 1983) se produce el traspaso de funciones y servicios de la Administración del Estado a la Comunidad Autónoma de Andalucía en materia de Educación.

Tras estas aclaraciones, se muestran las principales iniciativas legislativas y programáticas publicadas en los últimos años en relación con la biblioteca escolar en el contexto andaluz.

Tabla 1. Documentos legislativos y directrices de contexto andaluz sobre biblioteca escolar presentados en el presente apartado.

\begin{tabular}{|c|c|}
\hline $\begin{array}{l}\text { DIRECTRICES Y LEGISLACIÓN } \\
\text { CONTEXTO ANDALUZ }\end{array}$ & AÑo \\
\hline $\begin{array}{l}\text { Ley 16/2003, de } 22 \text { de diciembre, del Sistema Andaluz de Bibliotecas y Centros de } \\
\text { Documentación }\end{array}$ & 2003 \\
\hline $\begin{array}{l}\text { Acuerdo de } 23 \text { de enero de 2007, del Consejo de Gobierno, por el que se aprueba el } \\
\text { Plan de Lectura y de Bibliotecas Escolares en los Centros Educativos Públicos de } \\
\text { Andalucía }\end{array}$ & 2007 \\
\hline Ley $17 / 2007$, de 10 de diciembre, de Educación de Andalucía & 2007 \\
\hline Plan de trabajo y autoevaluación de la biblioteca escolar (2011) & 2011 \\
\hline Organización y funcionamiento de la biblioteca escolar (2011) & 2011 \\
\hline $\begin{array}{l}\text { Instrucciones de } 30 \text { de junio de } 2011 \text { de la Dirección General de Ordenación y } \\
\text { Evaluación Educativa sobre la organización y funcionamiento, durante el curso } \\
2011 / 2012 \text {, de las bibliotecas escolares de los centros docentes públicos que imparten } \\
\text { Educación Primaria o Educación Secundaria Obligatoria }\end{array}$ & 2011 \\
\hline Contribución de la biblioteca escolar al fomento de la lectura (2012) & 2012 \\
\hline $\begin{array}{l}\text { Programas para el desarrollo de la competencia informacional articulados desde la } \\
\text { biblioteca escolar (2012) }\end{array}$ & 2012 \\
\hline
\end{tabular}

Elaboración propia.

\section{Ley 16/2003, de 22 de diciembre, del Sistema Andaluz de Bibliotecas y Centros de Documentación}

En la "Exposición de motivos" se explica que la razón principal para promulgar esta Ley es la necesidad de actualizar el marco legislativo andaluz anterior contenido en la Ley $8 / 1983$, de 3 de noviembre, de Bibliotecas. Veinte años después no son pocos los cambios sociales y tecnológicos que conforman una realidad bien distinta, como explica el propio texto legal de 2003:

ISSN: 0212-8594 ISSN-e: 2340-2776 № DOI: http://dx.doi.org/10.12795/rea.2011.i28.02

REA 28 (2011):16-33

http://www.publius.us.es/estudios_andaluces 
La extensión de la educación formal y la generalización del acceso a los medios de comunicación han hecho de la cultura un concepto menos reverencial y más libre y, al propio tiempo, la sociedad andaluza ha incrementado su demanda en cuanto a calidad de vida y a la prestación de servicios. Las recientes tecnologías abren nuevas posibilidades para satisfacer tal demanda a través de los servicios bibliotecarios, poniendo al alcance de la mano el viejo deseo del Plan de María Moliner en la Segunda República Española: que cualquier ciudadano en cualquier lugar pueda disponer de cualquier registro cultural o de información.

El objetivo principal de esta Ley es, por tanto, ayudar a que los recursos bibliotecarios de Andalucía puedan estar al alcance de todos gracias, especialmente, a las nuevas tecnologías, y también al hecho de seguir las pautas internacionales y nacionales publicadas después de 1983.

En lo que se refiere a las bibliotecas escolares, la Ley aborda los siguientes aspectos:

- Definición. En el artículo 3 ofrece la siguiente: "La biblioteca escolar es la institución que reúne, organiza y pone a disposición de la comunidad escolar aquellos registros culturales y de información necesarios para el aprendizaje y el desarrollo personal de los escolares".

- Colaboración con otras instituciones. Como se explica en el artículo 9.3, las bibliotecas escolares podrán colaborar con las bibliotecas públicas de su zona incluso podrán formar parte de la Red de Bibliotecas Públicas de Andalucía- y también convenir con sus municipios la extensión de su uso a vecinos ajenos a la comunidad escolar.

- Sistema Andaluz de Bibliotecas y Centros de Documentación. Según lo establecido en el artículo 10, las bibliotecas escolares forman parte de este Sistema.

Acuerdo de 23 de enero de 2007, del Consejo de Gobierno, por el que se aprueba el Plan de Lectura y de Bibliotecas Escolares en los Centros Educativos Públicos de Andalucía

El objetivo primordial del Plan de Lectura y de Bibliotecas Escolares en los centros educativos públicos de Andalucía (en adelante Plan $L y B$ ) es potenciar el uso de las bibliotecas escolares con el fin crear oportunidades lectoras entre el alumnado.

Los contenidos del Plan LyB se estructuran del siguiente modo:

1. Introducción

1.1. Antecedentes

ISSN: 0212-8594 ISSN-e: 2340-2776 № DOI: http://dx.doi.org/10.12795/rea.2011.i28.02

REA 28 (2011):16-33

http://www.publius.us.es/estudios_andaluces 


\subsection{Justificación}

2. Líneas de actuación

2.1. Apoyo a la lectura y a las bibliotecas escolares

2.2. Desarrollo de proyectos lectores

2.3. Biblioteca virtual escolar de Andalucía

2.4. Programa de ediciones y publicaciones

3. La Formación del profesorado y el Plan LyB

4. El asesoramiento a los centros y la coordinación del Plan LyB

5. La evaluación del Plan LyB

Durante todo el texto se hace hincapié en la importancia de la biblioteca escolar no solo para el proceso de enseñanza-aprendizaje del alumnado, sino también para la vida del centro, como eje vertebrador del acceso a la información de toda la comunidad educativa.

Se trata de un proyecto sólido en el que se concretan las actuaciones que deben llevarse a cabo desde las distintas instancias para conseguir los objetivos propuestos. El Plan LyB se ha convertido en un instrumento eficaz que está dando sus frutos en Andalucía desde su implantación.

\section{Ley 17/2007, de 10 de diciembre, de Educación de Andalucía}

Esta Ley pretende revisar y actualizar las políticas educativas andaluzas a la luz de las estrategias marcadas por la Unión Europea en los primeros años del siglo XXI y también según las directrices establecidas para el ámbito español en la ya citada Ley Orgánica 2/2006, de 3 de mayo, de Educación. Pero sobre todo, la presente Ley de Educación de Andalucía promueve nuevos objetivos educativos, según el actual desarrollo social, y los medios que ayuden a hacer efectivo el derecho a la educación, ya que, como se explica en la "Exposición de motivos":

Las sociedades del conocimiento exigen más y mejor educación para todas las generaciones, elevar la calidad de los sistemas educativos, saberes más actualizados, nuevas herramientas educativas, un profesorado bien formado y reconocido, una gestión de los centros docentes ágil y eficaz, más participación y corresponsabilidad de las familias y demás agentes implicados, establecer nuevos puentes entre los intereses sociales y educativos y que las ventajas que de ello se deriven alcancen a toda la población, adoptando las medidas necesarias tanto para el alumnado con mayores dificultades de aprendizaje, como para el que cuenta con mayor capacidad y motivación para aprender.

Para lograr estos nuevos objetivos educativos, las bibliotecas escolares deben desempeñar un importante papel que, en nuestra opinión, no se refleja suficientemente en el contenido de esta Ley. Además de la utilización coordinada de las bibliotecas escolares y municipales (artículo 174), y de la inclusión de la biblioteca

ISSN: 0212-8594 ISSN-e: 2340-2776 № DOI: http://dx.doi.org/10.12795/rea.2011.i28.02

REA 28 (2011):16-33

http://www.publius.us.es/estudios_andaluces 
escolar en el reglamento de organización y funcionamiento de cada centro (artículo 128), solo se alude a este tipo de bibliotecas en el artículo 23 cuando se apunta que el profesorado jubilado podría colaborar en los planes de uso de las bibliotecas escolares y de animación a la lectura, aunque, eso sí, su tarea no podrá influir en la provisión de los puestos de trabajo establecidos.

\section{Plan de trabajo y autoevaluación de la biblioteca escolar (2011)}

Elaborado por García Guerrero para la Consejería de Educación de la Junta de Andalucía, es el primero de la serie denominada Documentos de referencia para bibliotecas escolares (DR1/BECREA). Son textos dirigidos a los docentes responsables de las bibliotecas escolares de los centros públicos de Andalucía para ayudarles a ejercer las responsabilidades y funciones encomendadas por la Consejería de Educación. El documento aborda los siguientes contenidos:

1. La biblioteca escolar en su contexto

1.1. Cometido y ámbitos de actuación de la biblioteca escolar en el Plan de Centro

1.2. ¿Qué elementos permitirán llevar a cabo y sostener las intervenciones en los distintos ámbitos de actuación de la biblioteca escolar?

1.3. El reconocimiento de la biblioteca que tenemos y el uso que hacemos de ella

- Situación A, primeros pasos

- Situación B, replanteamiento de servicios y usos

- Situación C, puesta en marcha de algunos servicios y programas

- Situación D, estabilidad y uso regular

2. Plan de trabajo de la biblioteca y autoevaluación

2.1. Los componentes para diseñar el "Plan de trabajo de la biblioteca escolar"

2.1.1. Los documentos directores de planificación e información

2.2. Aproximación a la evaluación de la biblioteca escolar

2.2.1. Cultura de evaluación

2.2.2. Hacia un modelo de evaluación

2.2.3. Dimensiones, indicadores y señales de avance

2.2.4. Procesos y ejemplificaciones:

1. Selección de las dimensiones e indicadores con sus señales de avance

2. Recogida de información

3. Análisis de la información y planificación

4. Registro de valoración general

5. Memoria anual e informe de evaluación

ISSN: 0212-8594 ISSN-e: 2340-2776 № DOI: http://dx.doi.org/10.12795/rea.2011.i28.02

REA 28 (2011):16-33

http://www.publius.us.es/estudios_andaluces 


\section{Organización y funcionamiento de la biblioteca escolar (2011)}

Elaborado por García Guerrero y Luque Jaime para la Consejería de Educación de la Junta de Andalucía, es el segundo de la serie denominada Documentos de referencia para bibliotecas escolares (DR2/BECREA). El documento aborda los siguientes contenidos:

1. Conocer qué biblioteca tenemos y saber qué biblioteca queremos

2. Distribuir tareas a los recursos humanos disponibles

3. Distribuir espacios, fondo y mobiliario

4. Señalizar espacios, áreas y secciones

5. Recopilar toda la documentación dispersa y llevarla (provisionalmente) a la biblioteca para realizar el proceso técnico

6. Realizar el primer expurgo

7. Separar y organizar los documentos librarios y no librarios

8. Realizar el segundo expurgo

9. Iniciar el proceso técnico (automatización con la aplicación Abies)

10. Forrar los volúmenes y reparar los documentos deteriorados

11. Definir la política de préstamo

12. Establecer la política documental (selección y adquisición de fondos)

13. Incorporar al Reglamento de Organización y Funcionamiento (ROF) las normas de uso de la biblioteca

14. Garantizar la presencia de la biblioteca escolar en el ciberespacio

15. Abrir la biblioteca y presentarla a la comunidad educativa

16. Planificación de actuaciones. El plan de trabajo de la biblioteca

Instrucciones de 30 de junio de 2011 de la Dirección General de Ordenación y Evaluación Educativa sobre la organización y funcionamiento, durante el curso 2011/2012, de las bibliotecas escolares de los centros docentes públicos que imparten Educación Primaria o Educación Secundaria Obligatoria

Como su nombre indica, se trata de un documento normativo que pretende unificar y concretar en los centros docentes públicos de Andalucía (Primaria y ESO) la organización y el funcionamiento de las bibliotecas escolares. Las instrucciones se refieren a los siguientes aspectos:

- Organización de la biblioteca escolar

- Persona responsable de la biblioteca escolar

- Funciones de la persona responsable de la biblioteca escolar

- Equipo de apoyo

- Certificación y reconocimiento

- Apertura de la biblioteca en horario extraescolar

- Acciones formativas

- Asesoramiento, seguimiento y evaluación

ISSN: 0212-8594 ISSN-e: 2340-2776 № DOI: http://dx.doi.org/10.12795/rea.2011.i28.02 
En este trabajo se incluyen únicamente estas instrucciones para el curso 2011-2012 porque son las más cercanas cronológicamente a la investigación realizada.

\section{Contribución de la biblioteca escolar al fomento de la lectura (2012)}

Elaborado por García Guerrero para la Consejería de Educación de la Junta de Andalucía, es el tercero de la serie denominada Documentos de referencia para bibliotecas escolares (DR3/BECREA). El documento aborda los siguientes contenidos:

1. La biblioteca escolar y el fomento de la lectura

1.1. Referencias y responsabilidades

1.2. No tanto la cantidad

1.3. Fomento de la lectura y educación literaria

1.4. Entornos para una lectura fomentada

2. Modelo de intervención desde la biblioteca escolar

2.1. Cuestión previa: Marco general sobre el tratamiento de la lectura en el centro

2.2. Coordinación y establecimiento de la política documental

2.2.1. Selección y adquisición

2.2.2. Coordinación y planificación de los itinerarios de lectura

2.2.2.1. Modelos de actuación para el fomento de la lectura literaria

2.2.3. Provisión de recursos librarios y en línea a través de la implementación de secciones específicas en las distintas aulas

2.3. Programa general desde la BECREA: coordinación y articulación de intervenciones y actividades de fomento de la lectura

2.4. Apoyo al tiempo reglado de lectura en todas las áreas y ámbitos y a los planes de lectura o proyectos lingüísticos

3. La biblioteca escolar y su entorno

3.1. Acción de colaboración

3.2. Política de lectura corresponsable. Zona educativa de cooperación bibliotecaria (ZECB)

4. Incorporación de las acciones de fomento y desarrollo de la lectura al Plan de trabajo de la BECREA y abordaje de la evaluación

5. Vinculación del responsable de la biblioteca escolar a la red profesional de su provincia

6. Algunos blogs de bibliotecas escolares con actuaciones de fomento de la lectura

7. Portales de referencia relacionados con el fomento de la lectura

ISSN: 0212-8594 ISSN-e: 2340-2776 № DOI: http://dx.doi.org/10.12795/rea.2011.i28.02

REA 28 (2011):16-33

http://www.publius.us.es/estudios_andaluces 


\section{Programas para el desarrollo de la competencia informacional articulados desde la biblioteca escolar (2012)}

Elaborado por Durban Roca, Cid Prolongo y García Guerrero para la Consejería de Educación de la Junta de Andalucía, es el cuarto de la serie denominada Documentos de referencia para bibliotecas escolares (DR4/BECREA). El documento aborda los siguientes contenidos:

1. La biblioteca escolar como recurso articulador de programas

1.1. Contribución de la biblioteca escolar al acceso y uso de la información

1.2. Proyectos de investigación y competencia informacional

2. Educación en información y competencias básicas

2.1. Competencia informacional y contenidos de la Ley Orgánica de Educación

2.1.1. Focalización y relación con las competencias

2.2. Sistematización de los contenidos implicados

2.2.1. Modelo de las tres fases

2.3. Corresponsabilidad de las áreas. Etapas de educación primaria y secundaria

3. Modelo de intervención desde la biblioteca escolar

3.1. Cuestión previa. Marco general para los programas formativos

3.2. Estructura y componentes de los programas formativos

3.3. Organización y vinculación de los programas a las áreas

3.4. Orientaciones metodológicas

3.4.1. Repertorio de propuestas metodológicas competenciales

4. Programa 1: Conocer la biblioteca y aprender a utilizarla. Programa formativo en el conocimiento de la biblioteca y los recursos que ofrece

5. Programa 2: Aprender a investigar y a utilizar la información. Programa formativo en habilidades y estrategias para aprender e informarse

6. Incorporación de las acciones para el desarrollo de la competencia informacional al Plan de trabajo anual de la biblioteca escolar y abordaje de la evaluación

7. Vinculación del responsable de la biblioteca escolar a la red profesional de su provincia

8. Portales de referencia sobre competencia informacional

ISSN: 0212-8594 ISSN-e: 2340-2776 № DOI: http://dx.doi.org/10.12795/rea.2011.i28.02 


\section{PROMOCIÓN DE LAS BIBLIOTECAS ESCOLARES EN ANDALUCÍA.}

En los últimos 20 años han sido muchas y muy variadas las iniciativas llevadas a cabo en Andalucía con el fin de promover las bibliotecas escolares así como de formar personas competentes en el uso eficiente de la información.

A continuación se muestran de manera resumida las principales iniciativas andaluzas en materia de biblioteca escolar que han servido de impulso y apoyo para convertirlas en protagonistas del centro educativo.

Se exponen las iniciativas agrupadas en torno a la siguiente tipología, y a su vez, se ordenan cronológicamente.
a) Instituciones, encuentros y actividades de formación
b) Publicaciones periódicas
c) Planes, proyectos y redes
d) Recursos y herramientas

Tabla 2. Contenidos sobre promoción de las bibliotecas escolares de contexto andaluz presentados en el presente apartado.

\begin{tabular}{l} 
PROMOCIÓN DE LAS BIBLIOTECAS ESCOLARES \\
CONTEXTO ANDALUZ \\
\hline Instituciones, encuentros y actividades de formación \\
\hline Congreso Andaluz de lectura "Leer para aprender" (2010) \\
\hline I Jornadas andaluzas de las redes profesionales de lectura y bibliotecas escolares (2011) \\
\hline Centro Andaluz de las Letras (CAL) \\
\hline Publicaciones periódicas \\
\hline Libro Abierto \\
\hline Planes, proyectos y redes \\
\hline Plan de Impulso de la Lectura en Andalucía (2011-2013) \\
\hline $\begin{array}{l}\text { Plan de Lectura y de Bibliotecas Escolares en los Centros Educativos Públicos de } \\
\text { Andalucía (Plan LyB) (2007) }\end{array}$ \\
\hline Averroes: la Red Telemática Educativa de Andalucía \\
\hline Redes Profesionales Provinciales de Lectura y Bibliotecas Escolares \\
\hline Recursos y herramientas \\
\hline Helvia. Plataforma educativa para los centros docentes de Andalucía \\
\hline Portal Biblioteca Escolar - Centro de Recursos para la Enseñanza y el Aprendizaje \\
\hline
\end{tabular}

Elaboración propia.

ISSN: 0212-8594 ISSN-e: 2340-2776 № DOI: http://dx.doi.org/10.12795/rea.2011.i28.02 


\subsection{INSTITUCIONES, ENCUENTROS Y ACTIVIDADES DE FORMACIÓN.}

\section{Congreso Andaluz de lectura "Leer para aprender" (2010)}

Este encuentro autonómico celebrado en Granada en 2010 tuvo una gran importancia para el desarrollo de las bibliotecas escolares en Andalucía. En el curso 2009-2010 se vio culminado el proceso de implementación del Plan de Lectura y Bibliotecas Escolares impulsado por la Consejería de Educación, y a partir de este congreso los coordinadores del Plan en cada centro pasaron a ser responsables de la biblioteca escolar apoyados por un equipo docente cualificado. García Guerrero explica los aspectos más destacables de este congreso en el preliminar del Plan de Trabajo y Autoevaluación de la biblioteca escolar:

Constituyó un hito para el desarrollo de las bibliotecas escolares andaluzas por cuanto a partir de este evento la Consejería de Educación acomete una política de generalización de aspectos nucleares con la finalidad de dar estabilidad de uso a todas las bibliotecas de los centros educativos públicos de primaria y secundaria. En el Congreso se significó la importancia de aprovechar el bagaje de conocimiento y experiencia acumulado durante la implementación del Plan de Lectura y Biblioteca Escolar y se subrayó la necesidad de dar pie a un ciclo que se caracterizase por la calidad y estabilidad del uso de la biblioteca escolar (García, J., 2011, p. 12).

\section{Jornadas andaluzas de las redes profesionales de lectura y bibliotecas escolares (2011)}

Organizadas por la Consejería de Educación de la Junta de Andalucía y celebradas en Nerja (Málaga), estas jornadas reunieron por primera vez a los responsables de las redes profesionales de lectura y bibliotecas de toda Andalucía, que a su vez tienen la función de establecer cauces de comunicación y participación entre los coordinadores del Plan de Lectura y Bibliotecas de las distintas provincias andaluzas.

\section{Centro Andaluz de las Letras (CAL) ${ }^{1}$}

Es un organismo dependiente de la Consejería de Cultura de la Junta de Andalucía adscrito a su Dirección General del Libro, Archivos y Bibliotecas. Dedica la mayor parte de su programación a la promoción de la lectura. Ha creado la Red Andaluza de Clubes de Lectura, en la que participan también bibliotecas escolares, así como el Circuito de Dinamización Lectora con el que apoya el desarrollo de actividades complementarias para los clubes de lectura. El CAL organiza periódicamente diversos tipos de sesiones formativas y convoca cada año el Premio CAL al Fomento de la Lectura. En 1998, 1999

\footnotetext{
${ }^{1}$ www.juntadeandalucia.es/cultura/caletras/opencms/es/portal.

ISSN: 0212-8594 ISSN-e: 2340-2776 № DOI: http://dx.doi.org/10.12795/rea.2011.i28.02

REA 28 (2011):16-33

http://www.publius.us.es/estudios_andaluces
} 
y 2000 organizó encuentros entre bibliotecarios y docentes para intercambiar experiencias e impulsar las bibliotecas escolares.

\subsection{PUBLICACIONES PERIÓDICAS.}

\section{Libro Abierto ${ }^{2}$}

Esta publicación, cuyo nombre completo es Libro Abierto de información y apoyo a las bibliotecas escolares de Andalucía, comenzó a editarse en el curso 2000-2001, primero en forma de boletín en papel y desde abril/mayo de 2010 (número 40) exclusivamente en edición digital y con periodicidad trimestral.

Surgió como apoyo a las bibliotecas escolares de la provincia de Málaga, pero poco a poco se fue transformando en una publicación de ámbito autonómico para apoyar a los responsables de organizar, sostener y coordinar los servicios, programas y actuaciones de las bibliotecas escolares.

\subsection{PLANES, PROYECTOS Y REDES.}

\section{Plan de Impulso de la Lectura en Andalucía (2011-2013)}

En el marco de Plan Estratégico para la Cultura en Andalucía (PECA) se aprobó en 2005 el I Primer Plan Integral para el Impulso de la Lectura (2005-2010), en el que se contemplaron las bibliotecas escolares como elementos dinamizadores esenciales en el ámbito educativo.

Su antecedente inmediato fue el Plan Andaluz de Fomento de la Lectura (2002-2004) que impulsó el desarrollo de las bibliotecas escolares como recurso con capacidad para articular proyectos globales de centro.

En su empeño por fomentar la actividad lectora entre los ciudadanos, el gobierno andaluz puso en marcha en 2011 el II Plan de Impulso de la Lectura en Andalucía, horizonte 2013.

Esta nueva versión del plan contempla los recientes cambios sociales y tecnológicos, así como la necesidad de integrar las distintas instancias institucionales con competencias en materia de lectura.

En el preámbulo del plan (2011, p. 5) se explica la importancia de la colaboración entre distintas instituciones, incluida la biblioteca escolar, para alcanzar el objetivo deseado:

\footnotetext{
${ }^{2}$ http://cepronda.org/libroabierto.

${ }^{3}$ www.juntadeandalucia.es/cultura/web/areas/letras/sites/consejeria/areas/letras/ II_plan_fomento_lectura.
}

ISSN: 0212-8594 ISSN-e: 2340-2776 № DOI: http://dx.doi.org/10.12795/rea.2011.i28.02 
Un Plan que adquiere una especial relevancia en tanto que se concibe desde una perspectiva integradora que busca la necesaria colaboración de otras instancias institucionales con competencias en materia de lectura e implica la coordinación de actuaciones para lograr el objetivo común del gobierno andaluz: mejorar los hábitos culturales de la ciudadanía y potenciar la lectura como elemento vertebrador del diálogo democrático.

\section{Plan de Lectura y de Bibliotecas Escolares en los Centros Educativos Públicos de Andalucía (Plan LyB) (2007)}

En el apartado 2.1.4 de esta investigación se incluyó este Plan al tratar las directrices y legislación sobre la biblioteca escolar en el contexto andaluz. Sin embargo, por la importancia de esta iniciativa para el impulso de las bibliotecas escolares en Andalucía, conviene destacar aquí algunos aspectos que la propia administración autonómica ha considerado logros relevantes del Plan. Es la actuación de mayor calado en Andalucía para el fomento de las bibliotecas escolares en toda su historia. A través de las actuaciones programadas y de la participación de los centros escolares se ha producido un importante cambio conceptual respecto a la función de la biblioteca escolar como centro de recursos de enseñanza y aprendizaje (BE/CREA) en el Plan de Centro y en la actividad docente.

En el apartado de antecedentes del II Plan de Impulso de la Lectura en Andalucía (2011, p. 10) se habla del Plan LyB en estos términos:

Si la puesta en funcionamiento de las bibliotecas escolares ha generado en los centros una mayor conciencia del valor del recurso bibliotecario, también ha permitido un incremento de uso de los servicios de préstamo, acceso a internet, lectura y consulta en sala, entre otros. Por otra parte, la política de promoción de la apertura de las bibliotecas de los centros educativos a la comunidad en horario extraescolar ha facilitado el acceso a los recursos informativos y de aprendizaje tanto al alumnado como a otros usuarios en franjas horarias amplias y ha permitido una acción compensadora. Desde la puesta en marcha del Plan LyB, un $35 \%$ de los centros han abierto sus bibliotecas en horario extraescolar.

Otros logros y actuaciones relevantes del Plan LyB han sido los siguientes:

- Dotaciones económicas a los centros públicos participantes para la adquisición y renovación de fondos bibliográficos.

- Avances en la organización y catalogación de la colección, en su automatización utilizando la aplicación ABIES (Aplicación para la automatización de la colección de las Bibliotecas Escolares del Ministerio de Educación).

ISSN: 0212-8594 ISSN-e: 2340-2776 № DOI: http://dx.doi.org/10.12795/rea.2011.i28.02

REA 28 (2011):16-33

http://www.publius.us.es/estudios_andaluces 
- Mejora de la infraestructura y el equipamiento de las bibliotecas escolares a través del Plan de Apertura de Centros.

- Edición de la colección Clásicos Escolares distribuida a todas las bibliotecas de los centros educativos públicos por convenio de colaboración suscrito entre la Consejería y la Asociación de Editores de Andalucía. Los textos de las obras se complementan con su correspondiente guía de propuestas didácticas para el trabajo en el aula.

\section{Averroes: Ia Red Telemática Educativa de Andalucía ${ }^{4}$}

Creada en 1996, es una red de centros educativos que usan internet como herramienta educativa, de información y de comunicación. Impulsada y mantenida por la Consejería de Educación, y renovada en el programa And@red, ofrece a la comunidad educativa contenidos relacionados con todas las áreas del currículo.

Como señala Ortiz, R. (2004), "la evolución de la aplicación de las tecnologías en los Centros Educativos ha pasado por diferentes momentos a lo largo de los años". Como antecedente de Averroes podemos señalar el Plan Andaluz de Introducción a la Informática (1986), conocido como Plan Alhambra, con dos objetivos fundamentales: la introducción de la informática en las distintas asignaturas del currículum y la enseñanza de la informática como una asignatura más.

Además de responder a las necesidades del profesorado, alumnado y familias, la Junta de Andalucía también pretende con Averroes evitar la discriminación que supondría para una parte del alumnado la imposibilidad de acceder a diversos recursos educativos a través de internet. Por ello, garantiza el acceso y utilización de las redes de comunicación en todos los centros educativos.

El proyecto Averroes también actúa sobre un aspecto esencial en la transformación y el uso de las nuevas tecnologías educativas: la formación del profesorado. Para ello ha contado con ayuda del Fondo Social Europeo.

La Red hace posible la comunicación y el intercambio de contenidos a través de internet entre los centros docentes andaluces de Educación infantil, primaria, secundaria, los centros de Enseñanza de Régimen Especial, centros de adultos, Equipos de Orientación Educativa y los 32 Centros del Profesorado (CEP) de la comunidad autónoma.

En la actualidad, además de diversos servicios (agenda, noticias, mapa de centros, etc.) la web de la Red cuenta con las siguientes secciones principales:

- Innovación e investigación

\footnotetext{
${ }^{4}$ www.juntadeandalucia.es/averroes.

ISSN: 0212-8594 ISSN-e: 2340-2776 № DOI: http://dx.doi.org/10.12795/rea.2011.i28.02

REA 28 (2011):16-33

http://www.publius.us.es/estudios_andaluces
} 
- Profesorado en red

- Formación del profesorado

- Recursos educativos

- Apoyo al currículo

\section{Redes Profesionales Provinciales de Lectura y Bibliotecas Escolares}

Con un mayor o menor desarrollo organizativo, las ocho provincias andaluzas están creando o cuentan ya con su propia Red Profesional de Lectura y Bibliotecas Escolares, un fenómeno nacido durante el curso 2004-2005 con las siguientes líneas y propuestas de actuación:

- Gestionar de manera colectiva el conocimiento surgido de la experiencia en este ámbito.

- Proponer actividades formativas presenciales y en línea.

- Ofrecer la participación en grupos de trabajo sobre diversos aspectos.

- Posibilitar el intercambio de ideas, informaciones y experiencias.

En definitiva, se trata de que los encargados de las bibliotecas escolares los centros de cada una de las provincias andaluzas cuenten no solo con una herramienta formativa e informativa, sino también con apoyo, interconexión, comunicación, asesoramiento y colaboración.

\subsection{RECURSOS Y HERRAMIENTAS.}

\section{Helvia. Plataforma educativa para los centros docentes de Andalucía ${ }^{5}$}

Desarrollada en software libre por la Consejería de Educación de la Junta de Andalucía, esta plataforma constituye una potente herramienta tecnológica a disposición de los centros TIC de Andalucía para:

- Organizar los contenidos curriculares, recursos y materiales didácticos.

- Planificar las tareas escolares y otras actuaciones docentes.

- Posibilitar la comunicación entre profesorado y alumnado del propio centro y con los de otros centros de la red de centros TIC.

Otras plataformas digitales también desarrolladas por la Consejería de Educación, como Séneca (gestión administrativa) o Pasen (comunicación con las familias), están dirigidas a un perfil concreto de usuario. Sin embargo, como se explica en el apartado correspondiente, Helvia combina la presentación de la página web del centro (sitio web) con la tutoría, programación y seguimiento de la tarea escolar, creación de recursos y materiales (aula virtual) y la publicación libre de un diario personal o

\footnotetext{
${ }^{5}$ www.juntadeandalucia.es/averroes/helvia/sitio.

ISSN: 0212-8594 ISSN-e: 2340-2776 № DOI: http://dx.doi.org/10.12795/rea.2011.i28.02 
comunicaciones públicas individuales o grupales (bitácora), desde una única entrada identificada.

Quizá la principal aportación de Helvia es su gestor de contenidos para que cada centro pueda contar con una web propia y manejarla de manera autónoma, incluso personalizar las secciones, sin necesidad de dominar la programación informática.

Además de una web accesible, también ofrece la posibilidad de sindicación de contenidos, de búsqueda y de edición de diversos formularios para recibir los comentarios de sus usuarios.

Para facilitar el aprendizaje del manejo de esta plataforma la Consejería de Educación ha publicado el documento Primeros pasos con Helvia en formato PDF.

\section{Portal Biblioteca Escolar - Centro de Recursos para la Enseñanza y el Aprendizaje ${ }^{6}$}

Este portal web ofrece información actualizada, recursos, enlaces y publicaciones sobre las bibliotecas escolares en Andalucía. Gestionado por la Delegación Provincial de la Consejería de la Junta de Andalucía en Málaga, su menú principal consta de los siguientes apartados:

- Normativa

- Organización y gestión

- ABIES

- Educación en información

- Fomento de la lectura

- Familia y lectura

- Acción cultural

\section{BIBLIOGRAFÍA}

Andalucía 2003, "Ley 16/2003, de 22 de diciembre, del Sistema Andaluz de Bibliotecas y Centros de Documentación", Boletín Oficial de la Junta de Andalucía, 31 de diciembre, [en línea]. Disponible en internet:..

http://www.uma.es/publicadores/eees/wwwuma/lau.pdf [Fecha de acceso 4 de mayo de 2012].

Andalucía 2007, "Acuerdo de 23 de enero de 2007, del Consejo de Gobierno, por el que se aprueba el Plan de Lectura y de Bibliotecas Escolares en los Centros Educativos Públicos de Andalucía", Boletín Oficial de la Junta de Andalucía, n. 29, 8 de febrero, pp. 8-15. Disponible también en internet:

http://www.juntadeandalucia.es/boja/boletines/2007/29/d/updf/d1.pdf [Fecha de acceso 4 de febrero de 2012].

\footnotetext{
${ }^{6}$ www.juntadeandalucia.es/averroes/bibliotecaescolar.

ISSN: 0212-8594 ISSN-e: 2340-2776 № DOI: http://dx.doi.org/10.12795/rea.2011.i28.02 
Andalucía 2007, “Acuerdo de 23 de enero de 2007, del Consejo de Gobierno, por el que se aprueba el Plan de Lectura y de Bibliotecas Escolares en los Centros Educativos Públicos de Andalucía", Boletín Oficial de la Junta de Andalucía, n. 29, 8 de febrero, pp. 8-15. Disponible también en Internet:

http://www.juntadeandalucia.es/boja/boletines/2007/29/d/updf/d1.pdf [Fecha de acceso 44 de febrero de 2012].

Andalucía 2007, "Ley 17/2007, de 10 de diciembre, de Educación de Andalucía", Boletín Oficial de la Junta de Andalucía, n. 252, 26 de diciembre, pp. 5-36. Disponible también en internet:

http://www.juntadeandalucia.es/boja/boletines/2007/252/d/updf/d1.pdf [Fecha de acceso 14 de marzo de 2012].

Andalucía 2010, "Decreto 328/2010, de 13 de julio, por el que se aprueba el Reglamento Orgánico de las Escuelas Infantiles de Segundo Ciclo, de los Colegios de Educación Primaria, de los Colegios de Educación Infantil y Primaria y de los Centros Públicos específicos de Educación Especial", Boletín Oficial de la Junta de Andalucía, n. 139, 16 de julio, pp. 34-57. Disponible también en internet:

http://www.juntadeandalucia.es/boja/boletines/2010/139/d/updf/d3.pdf [Fecha de acceso 15 de mayo de 2012].

Andalucía 2011, “Instrucciones de 30 de junio de 2011 de la Dirección General de Ordenación y Evaluación Educativa, sobre el tratamiento de la lectura durante el curso 2011/2012, para el desarrollo de la competencia en comunicación lingüística de los centros educativos públicos que imparten Educación Infantil, Educación Primaria y Educación Secundaria", Consejería de Educación, Dirección General de Ordenación y Evaluación Educativa, [en línea]. Disponible en Internet:

http://www.juntadeandalucia.es/averroes/bibliotecaescolar/images/MisPdf/normativ a/instrucciones30jun11lectura.pdf [Fecha de acceso 15 de noviembre de 2012].

Averroes: Red Telemática Educativa de Andalucía, [en línea]. Disponible en internet: http://www.juntadeandalucia.es/averroes/

Contribución de la biblioteca escolar al fomento de la lectura, Documento de Referencia para bibliotecas escolares DR3/BECREA. 2012, Junta de Andalucía, Consejería de Educación, Málaga, [en línea]. Disponible en internet: http://issuu.com/profejajm74/docs/dr3becrea [Fecha de acceso 14 de septiembre de 2012]

España 1978, "Constitución española", Boletín Oficial del Estado, n. 311, 29 de diciembre, pp. 29313-29424. Disponible en internet:

http://www.boe.es/aeboe/consultas/enlaces/documentos/ConstitucionCASTELLANO.p df [Fecha de acceso 12 de noviembre de 2011].

ISSN: 0212-8594 ISSN-e: 2340-2776 № DOI: http://dx.doi.org/10.12795/rea.2011.i28.02

REA 28 (2011):16-33

http://www.publius.us.es/estudios_andaluces 
España 1981, "Real Decreto 1075/1981, de 24 de abril, sobre Traspaso de Competencias, funciones y Servicios a la Junta de Andalucía en materia de Cultura", Boletín Oficial del Estado, n. 138, 10 de junio, pp. 13117-13121. Disponible también en internet:

http://travesia.mcu.es/portalnb/ispui/bitstream/10421/3062/1/And rd1075 1981.pdf [Fecha de acceso 7 de agosto de 2012].

España 1983, "Real Decreto 3936/1982, de 29 de diciembre, sobre traspaso de funciones y servicios de la Administración del estado a la Comunidad Autónoma de Andalucía en materia de educación", Boletín Oficial del Estado, n. 19, 22 de enero, pp. 1663-1664. Disponible también en internet:

http://www.boe.es/aeboe/consultas/bases datos/doc.php?id=BOE-A-1983-2324

[Fecha de acceso 9 de septiembre de 2012]

García Guerrero, José (2010): Utilidad de la biblioteca escolar: un recurso al servicio del proyecto educativo, Trea, Gijón.

Jiménez Fernández, C. M. a (2012): La información telemática de las bibliotecas escolares. El valor informativo y pedagógico de las webs de las bibliotecas de los centros de educación primaria de Andalucía y Extremadura. Tesis doctoral. Universidad de Salamanca.

Libro Abierto: publicación de información y apoyo a las Bibliotecas Escolares de Andalucía, [en línea]. Disponible en internet: http://cepronda.org/libroabierto

Organización y funcionamiento de la biblioteca escolar: tareas básicas: Documento de Referencia para bibliotecas escolares DR2/BECREA. 2011, Junta de Andalucía, Consejería de Educación, Málaga. Disponible en internet:

http://www.juntadeandalucia.es/averroes/bibliotecaescolar/images/MisPdf/DR2/DR2 BECREA.pdf [Fecha de acceso 31 de julio de 2012].

Plan de Lectura y de Bibliotecas escolares en los centros educativos de Andalucía. 2006, Junta de Andalucía, Consejería de Educación, Málaga.

Plan de trabajo y autoevaluación de la biblioteca escolar, Documento de Referencia para bibliotecas escolares DR1/BECREA. 2011, Junta de Andalucía, Consejería de Educación, Málaga, [en línea]. Disponible en internet:

http://www.juntadeandalucia.es/averroes/bibliotecaescolar/images/MisPdf/DR1/DR1 BECREA.pdf [Fecha de acceso 31 de julio de 2012].

Plataforma Educativa Helvia [en línea]. Disponible en internet: http://www.juntadeandalucia.es/averroes/helvia/sitio/

Yáguez, E. (2006): Guía práctica para el desarrollo y dinamización de la Biblioteca Escolar en Secundaria, Ministerio de Educación y Ciencia, Madrid.

ISSN: 0212-8594 ISSN-e: 2340-2776 № DOI: http://dx.doi.org/10.12795/rea.2011.i28.02

REA 28 (2011):16-33

http://www.publius.us.es/estudios_andaluces 